\title{
Spatially Resolved Forming Mechanisms Detection in Single Point Incremental Forming Using Synchrotron Based Texture Analysis
}

\begin{abstract}
MATEUS DOBECKI, ALEXANDER POECHE, and WALTER REIMERS
Despite the ongoing success of understanding the deformation states in sheets manufactured by single-point incremental forming (SPIF), the unawareness of the spatially resolved influence of the forming mechanisms on the residual stress states of incrementally formed sheet metal parts impedes their application-optimized use. In this study, a well-founded experimental proof of the occurring forming mechanisms shear, bending and stretching is presented using spatially resolved, high-energy synchrotron x-ray diffraction-based texture analysis in transmission mode. The measuring method allows even near-surface areas to be examined without any impairment of microstructural influences due to tribological reactions. The depth-resolved texture evolution for different sets of forming parameters offers insights into the forming mechanisms acting in SPIF. Therefore, the forming mechanisms are triggered explicitly by adjusting the vertical step-down increment $\Delta z$ for groove, plate and truncated cone geometries. The texture analysis reveals that the process parameters and the specimen geometries used lead to characteristic changes in the crystallites' orientation distribution in the formed parts due to plastic deformation. These forming-induced reorientations of the crystallites could be assigned to the forming mechanisms by means of defined reference states. It was found that for groove, plate and truncated cone geometries, a decreasing magnitude of step-down increments leads to a more pronounced shear deformation, which causes an increasing work hardening especially at the tool contact area of the formed parts. Larger step-down increments, on the other hand, induce a greater bending deformation. The plastic deformation by bending leads to a complex stress field that involves alternating residual tensile stresses on the tool and residual compressive stresses on the tool-averted side incrementally formed sheets. The present study demonstrates the potential of high-energy synchrotron x-ray diffraction for the spatially resolved forming mechanism research in SPIF. Controlling the residual stress states by optimizing the process parameters necessitates knowledge of the fundamental forming mechanism action.
\end{abstract}

https://doi.org/10.1007/s11661-021-06261-1

(C) The Author(s) 2021

\section{INTRODUCTION}

THE single-point incremental forming (SPIF) process enables the customized production of complexly shaped sheet parts for economical production of prototypes. ${ }^{[1]}$ Compared to conventional cold-forming methods, like deep drawing, the SPIF process is characterized by an increased forming limit and adaptable residual stress state. At present, an unfavorable residual stress distribution inhibits the use of SPIF-produced parts in

MATEUS DOBECKI, ALEXANDER POECHE, and WALTER REIMERS are with the Technische Universität Berlin - Insitute for Materials Science and Technology - Metallic Materials, Ernst-ReuterPlatz 1, 10587 Berlin, Germany. Contact e-mail: mateus.dobecki@tuberlin.de

Manuscript submitted 30 November 2020; accepted 21 March 2021.

Article published online April 20, 2021 fracture-critical applications because of their negative effect on fatigue strength.

According to the literature, the incrementally formed specimens in the residual stress states depend on the forming mechanisms at work. ${ }^{[2]}$ Three basic forming mechanisms have been reported: shearing, bending and stretching. ${ }^{[3]}$ However, a comprehensive study of the forming mechanism acting in SPIF via high-energy synchrotron $\mathrm{x}$-ray diffraction and their influence on the residual stress states is yet to be presented in the literature.

In the literature, divergent results regarding the main forming mechanisms in SPIF have been presented. Silva et al. ${ }^{[1]}$ Emmens and Boogaard ${ }^{[3]}$ assume a combination of stretching and bending as the major modes of deformation in the SPIF process and predict an additional stabilizing effect caused by these mechanisms as well. The authors conclude that the superposition of bending and stretching, referred to as bending under 
tension (BUT), enhances the samples' formability. In addition to BUT, the impact of shear was also discussed in both investigations. The authors emphasize the importance of distinguishing the shear modes into in-plane shear, shear over the sheet thickness and out-of-plane shear. ${ }^{[4]}$ However, for small step-down increments Sebastiani ${ }^{[5]}$ considers the influence of shear as a forming mechanism in the SPIF process to be negligible. Kim and Yang ${ }^{[6]}$ describe the through-thickness shear as the fundamental forming mechanism because of the similarity of the forming process in SPIF with metal spinning.

Maqbool and Bambach [7] confirm the through-thickness shear with their numerical simulation and emphasize its positive effect on geometrical accuracy, whereas Allwood et al. ${ }^{[8]}$ regard the through-thickness shear as the responsible mechanism for the increased forming limits in the SPIF process.

The influence of residual stresses on the application behavior of incrementally formed sheets was demonstrated in the investigations of Maaß et al.. ${ }^{[9]}$ In this study, the specimens' fatigue life under cyclic loading was significantly improved by an optimized residual stress state. However, the deformation process in SPIF is very complex so that the individual contribution of the forming mechanisms described above and their influence on the residual stress state are controversially discussed in the literature. The ongoing discussion is related to the current lack of experimental evidence on the forming mechanisms in SPIF. ${ }^{[10]}$ The experimental methods used to investigate the forming mechanisms in the SPIF process mainly refer to mechanical and optical experiments. At this point, knowledge about the effects of the forming mechanisms is based on phenomenological evaluations. A complete explanation of the deformation process and resulting residual stress state based on the mentioned forming mechanisms has not been reported yet. ${ }^{[2]}$

In recent years the texture analysis enables a profound understanding of plastic deformation mechanisms in forming processes. This way, Ray ${ }^{[11]}$ and Wassermann and Grewen ${ }^{[12]}$ reveal the evolution of texture during rolling of f.c.c. materials while Williams ${ }^{[13]}$ observes an impact of torsion testing on the resulting texture. These results are attributed to a specific texture evolution if material undergoes plastic deformation; thus, the texture analysis contains insights into the state of the deformation.

In regard to the first $\mathrm{x}$-ray diffraction analyses in the transmission mode of incrementally formed sheets by Maaß et al., ${ }^{[2]}$ the suitability of texture analyses for determining the forming mechanisms have been shown. Mishra et al. ${ }^{[14]}$ also use texture analysis to investigate the deformation mechanisms in SPIF using $x$-ray diffraction analyses in reflection mode. The authors examined the influences of the step-down increment, tool radius and wall angle on the texture evolution at the sheet thickness center. The authors observed that the impact of a step-down increment and tool radius on texture development is negligible. With the help of a visco-plastic self-consistent model, the authors detect through-thickness shear (TTS) as the primary forming mechanism in SPIF. However, the x-ray diffraction investigations' restriction to a small volume at the center of the sheet thickness does not include the full impact of all forming mechanisms on the forming process and resulting near-surface residual stress states.

This study uses a new experimental approach based on high-energy synchrotron $\mathrm{x}$-ray diffraction to detect the main forming mechanisms acting at the SPIF process. The latest improvements in synchrotron radiation sources generate highly brilliant radiation and high spatial resolutions simultaneously. Thus, it is possible to determine all fundamental forming mechanisms with spatial resolution using a single measuring method. In contrast to already performed x-ray diffraction experiments, ${ }^{[14]}$ the study's presented measuring method allows for in situ x-ray transmission experiments. This enables even near-surface areas to be examined without any impairment of microstructural influences due to tribological reactions and laborious preparations of the measured surface. This is important for understanding the process, since a pronounced texture evolution due to the forming mechanisms is observed, especially in the surface zones of the sheet metal.

The depth-resolved identification of the forming mechanism extends the findings of Mishra et al. [14] and allows statements to be made about their impact on the near-surface residual stress states.

SPIF is a plastic-forming process where, on the microstructural level, the forming mechanisms lead to characteristic changes of the spatial orientation distribution of the crystallites. ${ }^{[15]}$ Using depth-resolved high-energy synchrotron x-ray diffraction at the HEMS beamline P07 at PETRA-III (DESY, Hamburg, Germany), changes in crystallite orientations are spatially resolved as determined by analyzing the x-ray reflection intensities. ${ }^{[16]}$ Based on this analysis, pole figures are calculated. They represent a two-dimensional illustration of the texture, ${ }^{[6]}$ which is quantitatively described by the crystallites' three-dimensional orientation distribution function. ${ }^{[12]}$ From the pole figures, conclusions can be drawn about the major contributions of the forming mechanisms in the SPIF process. For the investigations presented here, samples made from the aluminum alloy 5083 were manufactured, adjusting the magnitude of the vertical step-down increment $\Delta z$ for the groove, plate and truncated cone geometries. To identify the major forming mechanisms in the SPIF process, pole figures were initially measured on reference specimens formed by bending or shear loads. These single-mode plastic deformations led to characteristic changes in the spatial orientation distribution of the crystallites. Subsequently, a comparison of the intensity distribution in the pole figures of the reference specimens and the SPIF-formed parts allowed the derivation of the occurring forming mechanisms in the observed gauge volume for a set of process parameters. To understand and optimize the residual stress states in incrementally formed metal parts, detailed insights regarding the forming mechanisms are necessary, which are obtained by the texture analysis described in the previous sentences. This way, the potential of the gained 
information provided by pole figures for the analysis of spatially resolved forming mechanisms in SPIF is explored for the first time to this extent.

\section{EXPERIMENTAL PROCEDURES}

\section{A. Manufacture of Specimen Geometries}

The specimens were incrementally formed on a five-axis CNC-milling machine (type: DMG MORI DMU50) with a free rotating forming tool at the Institute of Forming Technology and Lightweight Components, TU Dortmund University. The sheets made of aluminum alloy 5083 had an initial thickness of $t_{0}=1 \mathrm{~mm}$, dimensions of $230 \times 230 \mathrm{~mm}^{2}$ and $130 \times$ $130 \mathrm{~mm}^{2}$ (only for small cone geometry, respectively), an initial yield strength of $\sigma_{\mathrm{y}}=165 \mathrm{MPa}$ and an average grain size of $21 \pm 2 \mu \mathrm{m}$. A forming tool with a tool radius of $R_{\text {tool }}=7.5 \mathrm{~mm}$ and tool feed rate of $v_{f}=300$ $\mathrm{mm} / \mathrm{min}$ was used. Figure 1 provides a qualitative illustration of the basic components and parameters of the SPIF process.

For lubrication, the sheet metal surface was coated with a deep-drawing oil (Castrol Iloform PN 226) before starting the forming process. Linear, plate and truncated cone geometries were manufactured with a varying magnitude of step-down increments $(\Delta z)$ from 0.5 to 10 $\mathrm{mm}$ (see Table I; Figure 1). The number of increments $\left(\sum \Delta z\right)$ required is given by the component height $h$ and

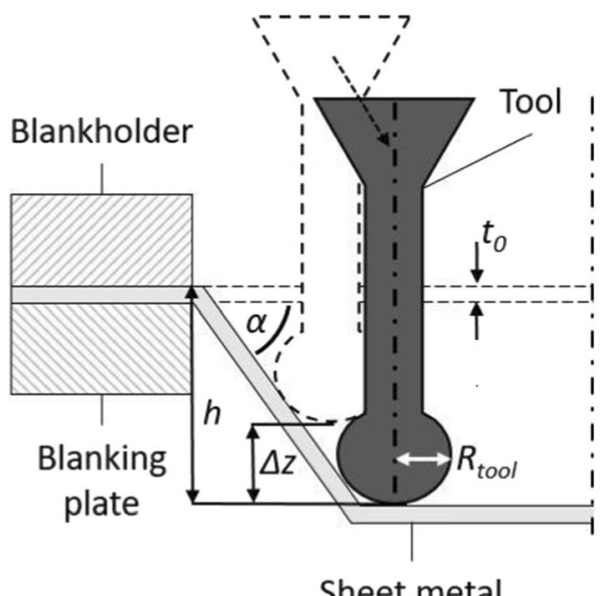

Fig. 1-Cross-sectional view of SPIF and its process parameters. the step-down increments used. The wall angle $\alpha$ was not varied for each geometry.

In addition to the initial sheet state, two further reference states were manufactured at the Institute of Forming Technology and Lightweight Components, TU Dortmund University: (1) a bent specimen, where the initial sheet was bent without lateral force to a $60 \mathrm{deg}$ angle in a free-bending process and (2) an in-plane torsion shear test referred to as a shear specimen.

An overview of the geometries produced and the locations investigated is shown in Figure 2.

\section{B. X-ray Diffraction Investigations}

To perform synchrotron x-ray diffraction experiments, the manufactured specimen geometries were cut into strips with dimensions $25 \times 1 \times 2 \mathrm{~mm}^{3}$. In Figure 2 the measuring area is highlighted blue for each geometry investigated. The described preparation of the specimens allows the implementation of synchrotron x-ray diffraction experiments in transmission mode. The synchrotron radiation beam is irradiating a specific gauge volume at the desired measuring location. These experiments were performed at the HEMS beamline P07 at PETRA-III at DESY (Hamburg, Germany), ${ }^{[1]}$ with the 2D detector Perkin Elmer XRD 1621. A hexapod realized the alignment of the samples. Setting the beam size to 300 $\mu \mathrm{m} \times 500 \mu \mathrm{m}$ by slits ensured that enough grains were irradiated to collect full diffraction rings. The $\mathrm{x}$-ray wavelength was set to $0.142347 \AA$ ( $E=87.1 \mathrm{keV})$. The hard $\mathrm{x}$-rays allow the investigation of material thicknesses of $2 \mathrm{~mm}$ without a significant loss of intensity for medium-absorbing materials. The diffraction image shows Debye fringes corresponding to the $h k l$ (Miller indices) reflections. The azimuthal variations of the $\mathrm{x}$-ray intensity indicate the preferred lattice orientations. The experimental setup is shown in Figure 3.

Lanthanum hexaboride was used as the calibration standard. The detector/image calibration was implemented with Fit2D software. ${ }^{[18]}$ The general procedure with the specimen analysis in MAUD (Material Analysis Using Diffraction) ${ }^{[19]}$ is based on integrating each image in slices, using 5 deg steps along the Debye fringes corresponding to 72 patterns per image. Using Rietveld refinement ${ }^{[20]}$ and the E-WIMV texture model, pole figures were calculated.

A pole figure is the two-dimensional projection of the occupancy ranges of a certain lattice plane from a considered plane direction (RD, TD, ND) of the specimen. The given intensities in the pole

Table I. Number and Magnitude of Step-Down Increments, Component Height and Specimen Geometries Used

\begin{tabular}{lccc}
\hline $\begin{array}{l}\text { Specimen } \\
\text { Geometry }\end{array}$ & $\begin{array}{c}\text { Component } \\
\text { Height }[\mathrm{mm}]\end{array}$ & Number of Forming Steps $\sum \Delta z /$ Step-Down Increment $\Delta z[\mathrm{~mm}]$ \\
\hline Linear roove & 10 & $10 / 1$ & $1 / 10$ \\
Plate & 10 & $0.5 / 20$ & $1.25 / 12$ \\
Small cone & 15 & $3.75 / 4$ & $3.75 / 9$ \\
Large cone & 33.75 & $5.625 / 6$ & \\
\hline
\end{tabular}



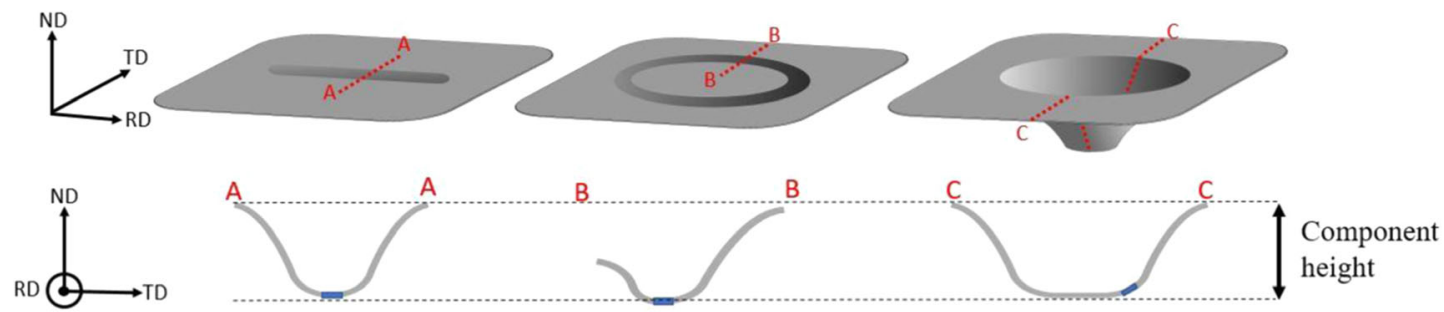
(a) cut A-A
(b) cut B-B
(c) cut C-C
Measuring position

Fig. 2-Schematic representation of the specimen geometries (a) linear groove; $(b)$ plate geometry; $(c)$ truncated cone geometry used and the analyzed measuring points for synchrotron x-ray diffraction (blue mark). RD represents the tool movement direction, TD the transverse to the toolpath direction and ND the sheet normal direction.

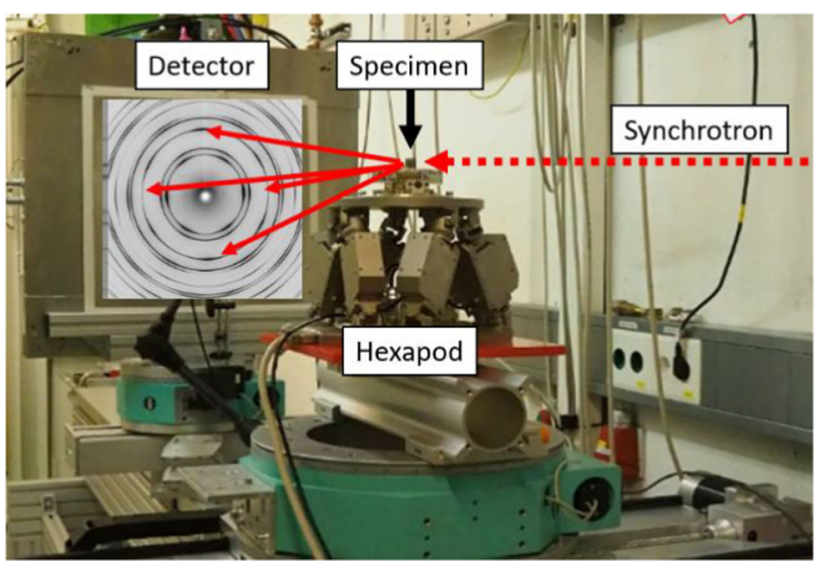

Fig. 3-Experimental setup at DESY; side-station EH1.

figures measure the occupancy density of the respective lattice planes' orientation. ${ }^{[12]}$ Since the maximum intensity of selected samples was found to be around 3.0 MRD (multiple of random distribution), the presentation of the here shown pole figure intensities was standardized to 3.0 MRD. All presented pole figures show the view from the sheet normal direction (ND).

Additionally, inverse pole figures for selected sample states have been calculated from the synchrotron x-ray diffraction measurements. Inverse pole figures are visualizations of selected sets of sample directions plotted concerning the crystal frame. In this work RD, TD and ND directions were used for the investigations. The sample directions are notated with $h k l$ (Miller indices), e.g. [100], [110] and [111]. The inverse pole figures are useful to illustrate certain types of textures. ${ }^{[12]}$

The residual stresses were measured by angle-dispersive $x$-ray diffraction $\left(\sin ^{2} \Psi\right.$ method) using monochromatic $\mathrm{Co}-\mathrm{K} \alpha$ radiation. In addition to the lattice spacing analysis, which is essential for determining residual stresses using the $\sin ^{2} \Psi$ method, the full width at half maximum (FWHM) was also determined at a sample tilt of $\psi=0$ deg. The FWHM is a measure for the present work-hardening state in the investigated volume for metallic materials. ${ }^{[21]}$ For the described angle-dispersive $\mathrm{x}$-ray diffraction analyses, aluminum reflection 311 was selected. Detailed information about the measurement

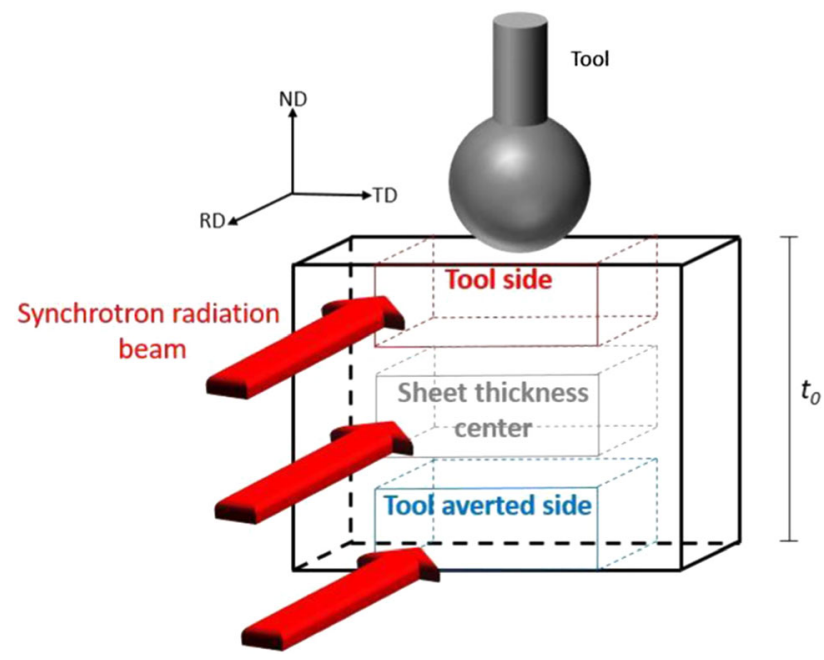

Fig. 4-Location of the gauge volumes over the sheet thickness.

setup and evaluation can be found in Maaß et al.. ${ }^{[2]}$ The determination of the near-surface residual stresses and FWHM was carried out for the two assumed principal directions in relation to the tool movement, $\sigma_{11}$ (rolling direction, RD) and $\sigma_{22}$ (transverse direction, TD).

\section{EXPERIMENTAL RESULTS}

\section{A. Reference Sample States}

To identify the main forming mechanism over the sheet thickness in SPIF by means of texture analysis, three reference sample states were selected: an initial sheet state (as delivered), a bent specimen and an in-plane torsion shear test. The spatial resolution of the high-energy synchrotron x-ray diffraction investigations is realized through three gauge volumes over the sheet thickness, illustrated in Figure 4. The gauge volumes are located at the tool side, sheet thickness center and tool-averted side from which the pole figures are determined in sheet normal direction.

From the gauge volumes, pole figures were determined for setting up the references for the intensity distribution of the forming mechanisms' bending, stretching and shear. Based on these defined intensity 
distributions, pole figures of the incrementally formed specimen are compared. The texture evolution incrementally formed specimens are assigned to the acting forming mechanisms in SPIF for a set of process parameters. The investigations showed that for the modified intensity distributions caused by plastic forming, the 220 pole figure is particularly suitable for determining changes due to bending and the 222 pole figure for shear deformation. All reference intensity distributions used are shown in Figure 5 by the pole figures.

As shown in Figures 5(a) and (b), the initial sheet reveals a fragile deformation texture due to cold rolling. ${ }^{[2]}$ This intensity distribution of the 222 pole figure (Figure 5(b)) can be, among other things, described by the ideal orientations (011)[21ㅍ] and (7 12 22) $[84 \overline{5}] .^{[12]}$ The ideal orientation is defined by the indication of the surface indices $(h, k, l)$ of the lattice plane and by the indices $[u, v, w]$ of the lattice direction in the chosen specimen orientation (RD, TD, ND). Compared to the texture of the initial sheet state, the 220 pole figure of the bending and 222 pole figure shear specimens (Figures 5(c) and (d)) indicate a significant change of the intensity distribution. Concerning the bend specimen, characteristic three intensity maxima on the pole figure's equatorial plane are recognizable at the tool-averted side. ${ }^{[2]}$ This described intensity distribution is assigned to plastic deformation by bending. It should be noted that the three maxima progressively lose intensity toward the tool side.

By contrast, the sheet deformed in an in-plane torsion test shows a distorted weak rolling texture and six newly developed intensity maxima at the edge of the 222 pole figure (see Figure 5d). The ideal orientation of the

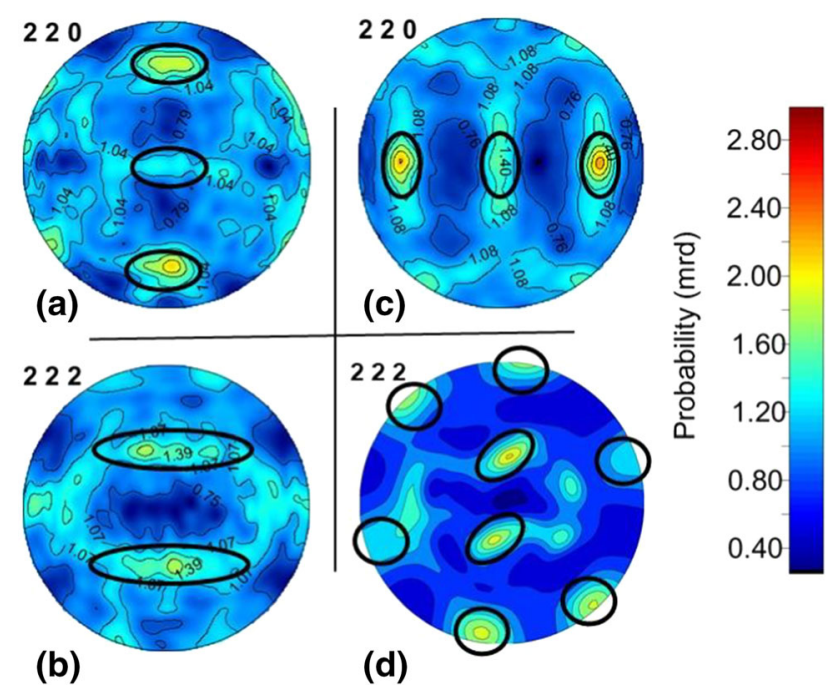

Fig. 5-Reference 220 and 222 pole figures and highlighted ideal orientations (black). ${ }^{[22]}$ (a) 220 pole figure: initial rolling texture (maximum intensity: $I_{\max }=1.8 \mathrm{MRD}$ ); $(b) 222$ pole figure: initial rolling texture $\left(I_{\max }=1.6 \mathrm{MRD}\right) ;(c) 220$ pole figure: bend specimen texture $\left(I_{\max }=2.6 \mathrm{MRD}\right) ;(d) 222$ pole figure: shear texture $\left(I_{\max }=2.8 \mathrm{MRD}\right)$. intensity maxima can be inter alia described as (111)

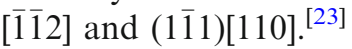

Inverse pole figures represent the direction-dependent orientation distribution of the crystal lattice planes in a fixed coordinate system relative to the sample. The characteristic orientation distributions of the crystal lattice planes of the bend specimen were examined to detect the influence of stretching on the plastic deformation.

This was done by taking advantage of the fact that a characteristic deformation state through the sheet thickness occurs because of bending deformation. Figure 6 shows inverse pole figures in the transverse tool path direction (TD) for the initial sheet state and the bend specimen on the tool and tool-averted side. The inverse pole figure of the bend specimen shows a significant increase in the 111 pole intensity on the tool-averted side. On the other hand, on the tool side of the sheet, a slight intensity maximum at the 110 pole occurred. This orientation of the lattice planes is in agreement with the elementary bending theory; according to that, the manifestation of the 111 pole is caused by bending-induced stretching and the 110 pole by bending-induced compression. ${ }^{[24]}$ The initial sheet state reveals a weak intensity maximum at the 111 pole on the tool and tool-averted side, according to sheet manufacturing by rolling. ${ }^{[12]}$

The following results are sorted according to the analyzed forming mechanism.

\section{B. Shear}

Texture responses due to shear deformation of incrementally (multi-stage) formed AA5083 sheets were studied with the aid of 222 pole figures on the tool side. Figure 7 illustrates selected 222 pole figures of linear groove and plate geometries with a total forming height of $10 \mathrm{~mm}$ and a varying number of step-down increments.

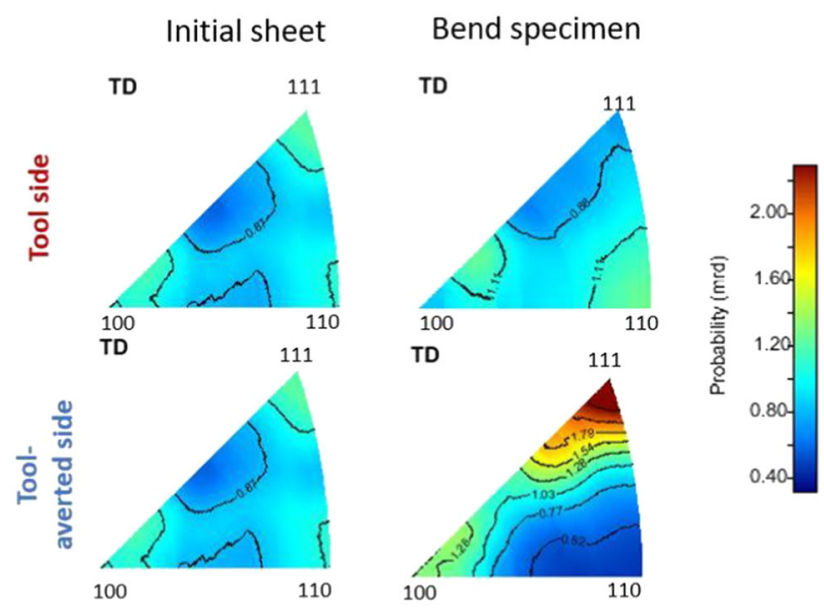

Fig. 6-Inverse pole figures of the initial sheet and bend specimen on the tool and tool-averted side; view direction: transverse tool movement direction (TD) ${ }^{[22]}$ 


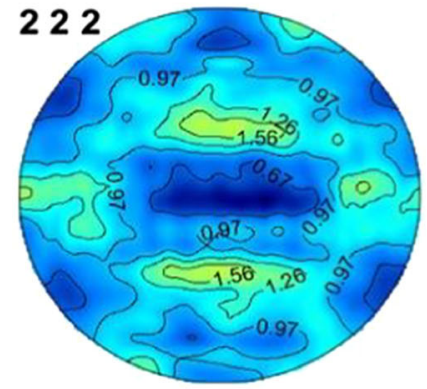

(a)

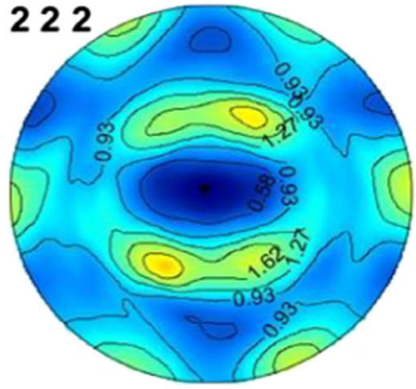

(b)
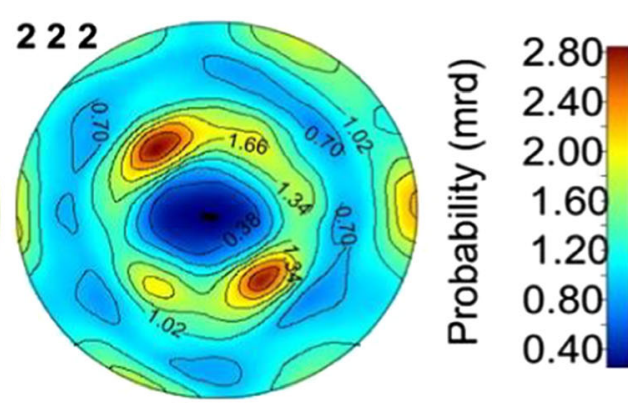

Fig. 7-Selected 222 pole figures on the tool side. (a) Single-stage incrementally formed linear groove $\left(\right.$ component height: $h=10$ mm), $\left(I_{\text {max }}=\right.$ $1.8 \mathrm{MRD}) ;(b)$ multi-stage incrementally formed linear groove with 10 step-down increments $(h=10 \mathrm{~mm}),\left(I_{\mathrm{max}}=2.5 \mathrm{MRD}\right)$; $(c)$ multi-stage incrementally formed plate geometry with 20 step-down increments $(h=10 \mathrm{~mm}),\left(I_{\max }=2.9 \mathrm{MRD}\right)$.

Figure 7a presents the 222 pole figure of a single-stage incrementally formed linear groove.

The intensity distribution can be attributed to the rolling texture (see Figure 5(b)). Comparing the maximum intensity of the rolling textures (Figures 5(b) and $7(\mathrm{a})$ ), the single-stage incremental forming process leads to an increase in intensity to $1.8 \mathrm{MRD}$. The influence of a multi-stage formed linear groove on the intensity distribution in the 222 pole figure is shown in Figure 7(b)). After ten step-down increments, a slight distortion of the initial rolling texture and the formation of six further intensity maxima at the edge of the pole figure can be observed. This intensity distribution qualitatively corresponds to the reference shear texture in Figure 7(d). This texture development caused by multi-stage plastic forming can be seen significantly for the plate geometry using 20 step-down increments shown in Figure 7c.

In Figure 8, 222 pole figures of truncated small (a) and large (b) cone geometries on the tool side with the maximum number of step-down increments used for each geometry are illustrated. Using the maximum number of step-down increments $\left(\sum \Delta z=12 ; \Delta z=\right.$ $1.25 \mathrm{~mm}$ ) for the small truncated cone geometry, the influence of through-thickness shear on the texture development during plastic forming is evident. In contrast to this, the maximum number of step-down increments $\left(\sum \Delta z=18 ; \Delta z=1.875 \mathrm{~mm}\right)$ used for manufacturing large cone geometries does not show a distinct influence of shear deformation in the intensity distribution in the 222 pole figure. This can be deducted from the missing occurrence of six characteristic intensity maxima at the edge of the 222 pole figure. The initial rolling texture is preserved after the forming process. An influence of the remaining shear modes (in- and outof-plane shear) has not been observed in the presented investigations.

\section{Bending}

The forming of linear, plate and truncated cone geometries causes a texture development influenced by bending deformation on the sheets' tool-averted side. Therefore, the bending-induced changes of the intensity distribution in the 220 pole figures are particularly

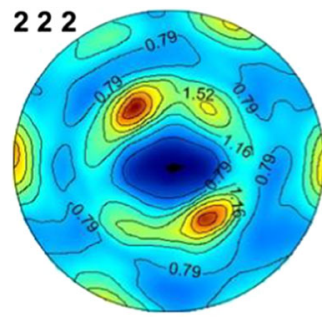

(a)
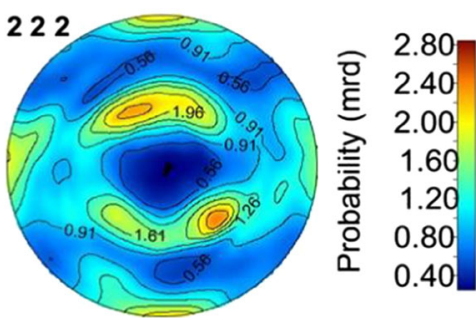

(b)
Fig. 8-Selected 222 pole figures on the tool side: (a) multi-stage incrementally formed small truncated cone geometry with 12 step-down increments $(h=15 \mathrm{~mm}),\left(I_{\max }=2.6 \mathrm{MRD}\right){ }^{[22]} ;(b)$ multi-stage incrementally formed large truncated cone geometry with 9 step-down increments $(h=33.75 \mathrm{~mm}),\left(I_{\max }=2.5 \mathrm{MRD}\right)$.

pronounced for increasing step-down increments and a reduced number of step-down increments, respectively. Figure 9 shows selected 220 pole figures of linear and plate geometries manufactured with a total forming height of $h=10 \mathrm{~mm}$. The presented results show an increasing bending influence on the intensity distribution in the 220 pole figures for single-stage formed linear grooves due to the high plastic deformation (Figure 9(a)). In this respect, three new intensity maxima developed on the equatorial plane of the 220 pole figure with a maximum intensity $I_{\max }$ of $1.9 \mathrm{MRD}$. The intensity distribution is comparable to the 220 pole figure of the bend specimen (see Figure 5(c)). Although the intensity distribution in the 220 pole figure changes due to bending at the near-surface tool-averted side, the initial rolling texture is still preserved in an attenuated form with a maximum intensity of $1.3 \mathrm{MRD}$. In an opposing trend, a decreasing vertical step-down increment causes a texture development without a significant influence of bending deformation on the sheets' tool-averted side. Regarding this process parameter for multi-stage formed linear grooves and plate geometries, no characteristic bending-induced intensity distribution can be observed in the 220 pole figures (see Figures 9(b) and (c)).

The 220 pole figures of small (a) and large (b) truncated cone geometries on the tool-averted side with the minimum number of step-down increments used for 


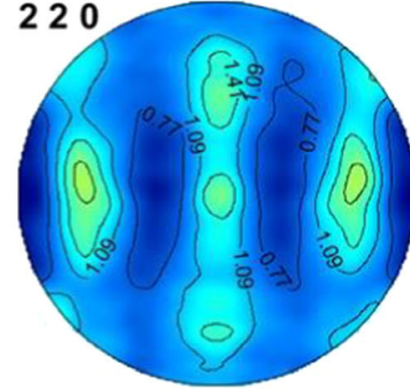

(a)

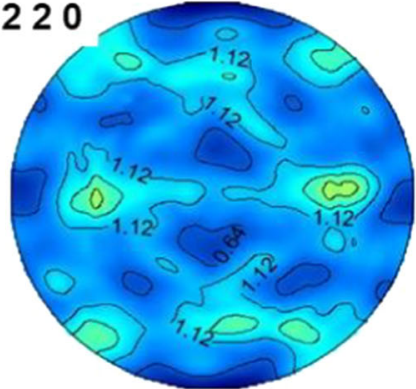

(b)
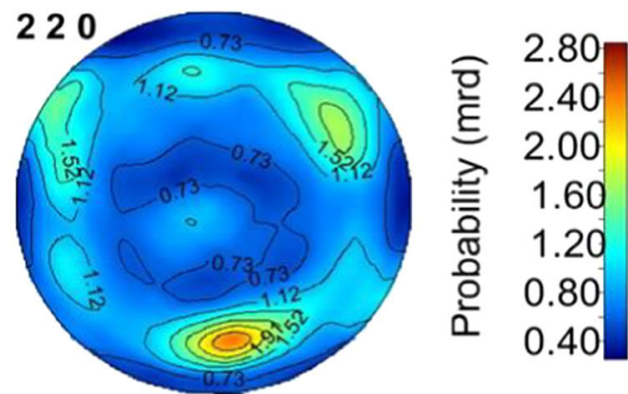

Fig. 9-Selected 220 pole figures on the tool-averted side: $(a)$ single-stage incrementally formed linear groove $(h=10$ mm $),\left(I_{\text {max }}=2.0 \mathrm{MRD}\right)$; (b) multi-stage incrementally formed linear groove with 10 step-down increments $(h=10 \mathrm{~mm})$, $\left(I_{\max }=1.9 \mathrm{MRD}\right)$; $(c)$ multi-stage incrementally formed plate geometry with 20 step-down increments $(h=10 \mathrm{~mm}),\left(I_{\max }=2.6 \mathrm{MRD}\right)$.

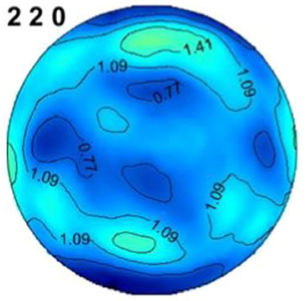

(a)

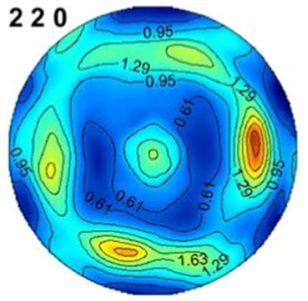

(b)

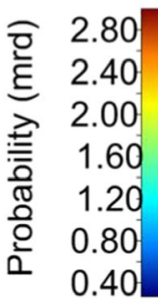

Fig. 10-Selected 220 pole figures on the tool-averted side: ${ }^{[22]}(a)$ Multi-stage incrementally formed small truncated cone geometry with 4 step-down increments $(h=15 \mathrm{~mm}),\left(I_{\max }=1.6 \mathrm{MRD}\right) ;(b)$ multi-stage incrementally formed large truncated cone geometry with 6 step-down increments $(h=33.75 \mathrm{~mm}),\left(I_{\max }=2.7 \mathrm{MRD}\right)$.

each geometry are shown in Figure 10. Different texture development depending on process parameters can be seen in the figure for the two types of truncated cones. For the large truncated cone geometry $\left(\sum \Delta z=6 ; \Delta z=\right.$ $5.625 \mathrm{~mm}$, Figures 10(b)), a significant influence of bending deformation on the intensity distribution was determined.

This is demonstrated by the formation of three intensity maxima on the equatorial plane, which can be assigned to bending deformation. The initial rolling texture is still present, but the ideal orientations were distorted through the sheet's thickness. The 220 pole figure for the small cone geometry $\left(\sum \Delta z=4 ; \Delta z=3.75\right.$ $\mathrm{mm}$ ) is illustrated in Figure 10a. Contrary to the observations in Figure 10b, the lowest number of step-down increments used for the small cone geometry does not lead to a formation of any characteristic intensity maxima due to bending deformation on the near-surface tool-averted side. The only common aspect towards Figure 10b is the slight distortion of the rolling texture.

\section{Stretching}

The inverse pole figure in transverse direction to the tool path on the tool-averted side of the single-stage formed linear groove $(\Delta z=10 \mathrm{~mm})$ (see Figure 11) shows a similar intensity distribution as the bend specimen (see Figure 6) on the tool-averted side.

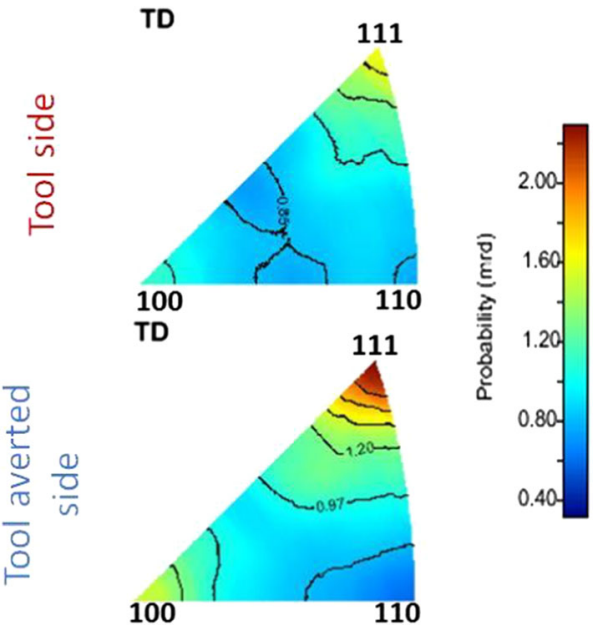

Fig. 11-Inverse pole figures of a single stage formed linear groove $(\Delta z=10 \mathrm{~mm})$ on the tool and tool-averted side; view direction: transverse tool movement direction (TD).

Therefore, the intensity of the 111 pole is particularly pronounced and is due to stretching of the sheet caused by bending (see subsection 3.1). On the tool side, in turn, an intensity maximum can be noticed at the 111 pole for the linear groove presented. This means that in contrast to the bend specimen, stretching is also present on the tool side of incrementally formed specimens. Therefore, the bending mechanism is superimposed with a global stretching in SPIF. There is no indication of compression on the tool side for incrementally formed specimens due to bending in the measurement direction under consideration. It is worth noting that the impact of stretching became more apparent in texture analysis with larger step-down increments $\Delta z$.

\section{E. Residual Stress}

The results concerning the angle-dispersive $\mathrm{x}$-ray diffraction analyses are presented in this section.

While Table II contains residual stresses and FWHM values and thus delivers information about the reference sample states, Tables III and IV demonstrate the impact of the SPIF process for various geometries on the 
Table II. Near-Surface Residual Stresses and FWHM on the Tool and Tool-Averted Side of the Reference Specimens

\begin{tabular}{|c|c|c|c|c|c|}
\hline Measuring Location & Measuring Direction & Measured Variable & Initial Sheet & Shear Specimen & Bend Specimen \\
\hline \multirow[t]{2}{*}{ Tool side } & $\mathrm{RD}$ & $\begin{array}{l}\sigma_{11}[\mathrm{MPa}] \\
\text { FWHM [deg] }\end{array}$ & $\begin{array}{l}5 \pm 10 \\
0.61\end{array}$ & $\begin{array}{c}-15 \pm 10 \\
0.71\end{array}$ & $\begin{array}{c}30 \pm 10 \\
0.62\end{array}$ \\
\hline & TD & $\begin{array}{l}\sigma_{22}[\mathrm{MPa}] \\
\text { FWHM [deg] }\end{array}$ & $\begin{array}{c}5 \pm 10 \\
0.61\end{array}$ & $\begin{array}{c}30 \pm 10 \\
0.71\end{array}$ & $\begin{array}{c}130 \pm 10 \\
0.64\end{array}$ \\
\hline \multirow[t]{2}{*}{ Tool-averted side } & $\mathrm{RD}$ & $\begin{array}{l}\sigma_{11}[\mathrm{MPa}] \\
\text { FWHM [deg] }\end{array}$ & $\begin{array}{c}-5 \pm 10 \\
0.60\end{array}$ & $\begin{array}{c}20 \pm 15 \\
0.71\end{array}$ & $\begin{array}{c}-35 \pm 10 \\
0.70\end{array}$ \\
\hline & TD & $\begin{array}{l}\sigma_{22}[\mathrm{MPa}] \\
\text { FWHM [deg] }\end{array}$ & $\begin{array}{c}5 \pm 10 \\
0.61\end{array}$ & $\begin{array}{c}-20 \pm 15 \\
0.72\end{array}$ & $\begin{array}{c}-70 \pm 10 \\
0.72\end{array}$ \\
\hline
\end{tabular}

Table III. Near-Surface Residual Stresses and FWHM on the Tool and Tool-Averted Side of Incrementally Formed Grooves and Plate Geometries

\begin{tabular}{|c|c|c|c|c|c|}
\hline \multirow[b]{2}{*}{ Measuring Location } & \multirow[b]{2}{*}{ Measuring Rection } & \multirow[b]{2}{*}{ Measured Variable } & \multirow{2}{*}{$\begin{array}{l}\text { Linear Groove } \\
10 / 1\end{array}$} & \multirow{2}{*}{$\begin{array}{c}\text { Linear Groove } \\
\Delta z / \sum \Delta z \\
1 / 10\end{array}$} & \multirow{2}{*}{$\begin{array}{c}\text { Plate } \\
0.5 / 20\end{array}$} \\
\hline & & & & & \\
\hline \multirow[t]{3}{*}{ Tool side } & $\mathrm{RD}$ & $\begin{array}{l}\sigma_{11}[\mathrm{MPa}] \\
\text { FWHM [deg] }\end{array}$ & $\begin{array}{c}-5 \pm 12 \\
0.63\end{array}$ & $\begin{array}{c}25 \pm 20 \\
0.75\end{array}$ & $\begin{array}{c}40 \pm 10 \\
0.81\end{array}$ \\
\hline & $\mathrm{TD}$ & $\sigma_{22}[\mathrm{MPa}]$ & $125 \pm 15$ & $6 \pm 20$ & $20 \pm 10$ \\
\hline & RD & $\begin{array}{l}\text { FWHM [deg] } \\
\sigma_{11}[\mathrm{MPa}]\end{array}$ & $\begin{array}{c}0.65 \\
-200 \pm 20\end{array}$ & $\begin{array}{c}0.73 \\
-220 \pm 20\end{array}$ & $\begin{array}{c}0.81 \\
-230 \pm 20\end{array}$ \\
\hline \multirow{3}{*}{ Tool-averted side } & & FWHM [deg] & 0.67 & 0.68 & 0.78 \\
\hline & TD & $\sigma_{22}[\mathrm{MPa}]$ & $-140 \pm 15$ & $-85 \pm 10$ & $-45 \pm 10$ \\
\hline & & FWHM [deg] & 0.68 & 0.67 & 0.76 \\
\hline
\end{tabular}

mentioned measured variables. It should be noted that the maximum deviation of the FWHM is $0.02 \mathrm{deg}$ for all investigated specimens shown here.

The residual stresses and the FWHM of the reference sample states are presented in Table II. Therein, the initial sheet state shows minor residual stresses and less pronounced FWHM values (0.61 deg) in every measuring direction on the tool as well as on the tool-averted side. In contrast to this, the shear specimen reveals a significant increase in the FWHM value to $0.71 \mathrm{deg}$ on the tool and tool-averted side. However, the plane residual stress state remains almost unaffected by the shear deformation. In an opposing trend, the bent specimen shows a specific residual stress state, characterized by residual tensile stresses on the tool and residual compressive stresses on the sheet's tool-averted side.

This characteristic residual stress state results from the relaxation of the bending-induced compressive stresses on the tool side and tensile stresses on the tool-averted side after the free-bending process. It is worth noting that the residual stress components perpendicular to the bending axis (TD, $\sigma_{22}$ ) are higher than the longitudinal residual stress components $\left(\mathrm{RD}, \sigma_{11}\right)$. It turns out that the FWHM of the bent specimen is more pronounced on the tool-averted (FWHM $=0.72 \mathrm{deg}$ ) than on the tool side (FWHM $=0.63 \mathrm{deg}$ ) of the reference sheet state caused by a stronger bending-induced plastic deformation with increasing distance to the tool contact. ${ }^{[2]}$

Table III shows the results of the angle-dispersive $\mathrm{x}$-ray diffraction measurements containing the residual stresses and FWHM of incrementally formed linear grooves and plate geometries. The data in the Table III indicate that forming a groove by a single forming step $\left(\Delta z=10 \mathrm{~mm} / \sum \Delta z=1\right)$ causes pronounced tensile residual stresses on the tool side, primarily transverse to the tool movement direction, and residual compressive stresses on the tool-averted side. Due to the single forming process, the tool-averted side has a more pronounced FWHM than the tool side of the sheet. Generally, this effect is more evident using major incremental step sizes. An increase of the forming steps to 10 by retaining the component height leads to a noticeable reduction of the $\sigma 22$ residual stress components on the tool's and tool-averted side of the formed linear groove. The most significant influence of a growing number of forming steps was observed by an increase in FWHM on the tool side $(0.75 \mathrm{deg})$ to a much greater extent than the tool-averted side $(0.67 \mathrm{deg})$. The tendency described can be seen more clearly in the plate geometry shown, formed with 20 forming steps. Since FWHM values represent the degree of work hardening that has been induced during forming, higher FWHM values on the tool side imply a stronger hardening of the tool contact area in the case of multiple formed geometries. 
The residual stresses and FWHM on the tool-averted side of small and large cone geometries are presented in Table IV. It is to be noted that both cone geometries are formed with an identical step-down increment $(\Delta z=$ $3.75 \mathrm{~mm}$ ) and distinguish in the component height. Generally, for both geometries, residual compressive stresses were evaluated from XRD measurements. Nevertheless, noticeable deviations in the magnitudes of these stresses can be observed between the sample states. In this regard, higher residual compressive stresses for large cones, especially in the transverse direction of tool movement (TD, $\sigma_{22}$ ), compared to the small cone geometry, can be taken from Table IV. The FWHM values are more pronounced for the larger cone with $0.74 \mathrm{deg}$ than $0.70 \mathrm{deg}$ for the small cone geometry.

\section{DISCUSSION}

\section{A. Shear}

To understand the role of shear deformation in the SPIF process, the analysis of textures is particularly well suited. The texture measurements revealed crystal reorientations due to shear, especially on the near-surface tool side of the specimen (see Figs. 5, 7 and 8). This is due to the direct tool contact. The analysis carried out points to a dependence of the occurring shear deformation on the magnitude and number of the vertical step-down increments $\Delta z$ used and the sheet's present hardening state.

The intensity of the initial rolling texture increases on the near-surface tool side of linear grooves with a single $10-\mathrm{mm}$ step-down increment. This is caused by the tool's forming force and the low hardening state of the initial sheet (see Table II). Therefore, the tool causes a near-surface roll-like deformation, which is the reason for the preservation and intensification of the rolling texture in the 222 pole figure shown in Figure 7(a). The roll-like deformation is characterized by stretching in the rolling direction and a compressive force in normal direction. ${ }^{[12]}$ The analysis of inverse pole figures in rolling and normal direction on the near-surface tool side confirms the roll-like deformation exerted by the tool for the single-stage incrementally formed linear groove on the near-surface tool side. This observation was more pronounced using larger step-down increments (no figure shown here).

During SPIF, the punch passes the gauge volume under consideration several times using decreasing step-down increments. Each step increases the dislocation density and leads to significant hardening of the tool contact area (see Table III). The work hardening tends to increase more strongly on the near-surface tool side than on the near-surface tool-averted side with a higher number of step-down increments used.

This observation can be demonstrated by the example of linear grooves presented in Table III. It is important to note that an increasing dislocation density mainly causes peak-broadening (FWHM) during plastic deformation. In this regard, an increase in FWHM, and therefore pronounced work hardening, on the near-surface tool side from $0.63 \mathrm{deg}$ for single-stage formed to $0.75 \mathrm{deg}$ for a multi-stage formed groove $\left(\sum \Delta z=10\right)$ can be observed. It should be noted that both linear grooves have the same component height $(h=10 \mathrm{~mm})$.

The increasing work hardening at the tool contact area using multiple forming steps is caused by increasing shear dominance, which is observed in the 222 pole figures with an increased number of step-down increments used (see Figures 7(b) and (c)). During the first forming steps, the material hardens most strongly. ${ }^{[25]}$ During forming, as the part is manufactured step by step, defects and dislocations accumulate, particularly at the tool contact area of the part, and cause a progressive hardening towards a steady-state range. At this point, the roll-like deformation caused by single-stage forming
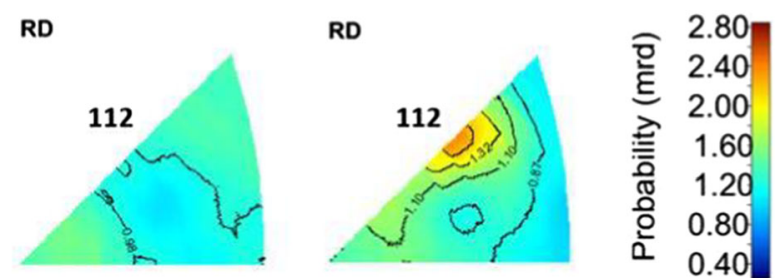

(a) sheet thickness center

(b) near-surface tool side

Fig. 12-Inverse pole figures of a linear groove $\left(\sum \Delta z=20\right)$ at the sheet thickness center $(a)$ and tool side $(b)$; view direction: tool movement direction (RD).

Table IV. Near-Surface Residual Stresses and FWHM on the Tool/Tool-Averted Side of Incrementally Formed Truncated Cone Geometries ${ }^{[22]}$

\begin{tabular}{|c|c|c|c|c|}
\hline \multirow[b]{2}{*}{ Measuring Location } & \multirow[b]{2}{*}{ Measuring Direction } & \multirow[b]{2}{*}{ Measured Variable } & \multicolumn{2}{|c|}{$\begin{array}{l}\text { Small Cone } \\
\qquad \Delta z / \sum \Delta z\end{array}$} \\
\hline & & & $3.75 / 4$ & $3.75 / 9$ \\
\hline Tool-averted side & $\begin{array}{l}\mathrm{RD} \\
\mathrm{TD}\end{array}$ & $\begin{array}{l}\sigma_{11}[\mathrm{MPa}] \\
\text { FWHM [deg] } \\
\sigma_{22}[\mathrm{MPa}] \\
\text { FWHM [deg] }\end{array}$ & $\begin{array}{c}-155 \pm 10 \\
0.71 \\
-50 \pm 10 \\
0.70\end{array}$ & $\begin{array}{c}-130 \pm 20 \\
0.74 \\
-180 \pm 15 \\
0.74\end{array}$ \\
\hline
\end{tabular}


is replaced by an increasing shear deformation mode, as further plastification is only possible through shear in the increased hardening state. ${ }^{26]}$

With increasing forming steps to 20, stronger shear influences the intensity distribution in the 222 pole figure (see Figure 7(c)) and leads to a significant increase in the FWHM from $0.61 \mathrm{deg}$ of the initial sheet state to $0.81 \mathrm{deg}$ for the multi-stage formed plate geometry (see Tables II and III).

The locally intensified shear at the tool side is quantified in the numerical simulations of Malhotra et al.. ${ }^{[27]}$ For determining the type of shear acting in SPIF, inverse pole figures in rolling direction for the near-surface tool side and the sheet thickness center are illustrated in Figure 12. To examine the influence of repetitive tool movement on the material, an additional stretching gradient over the sheet thickness was measured in the tool movement direction. Using inverse pole figures, an elongation of the material caused by the tool movement in the rolling direction at the near-surface tool side was detected (see Figure 12). The intensity distributions show a pronounced 112 pole at the near-surface tool side (Figure 12(b)). The 112 pole occurs because of elongation for f.c.c. metals. ${ }^{[24]}$

However, the elongation only occurred in an attenuated form in the middle of the sheet thickness (Figure 12(a)). This way, the difference in elongation between the near-surface tool side and the middle of the sheet thickness supports the hypothesis of through-thickness shear (TTS). Furthermore, it was proven with the $\sin ^{2} \Psi$ method that a $\Psi$-splitting could only be observed in the longitudinal section of the sample perpendicular to the sheet surface (no figure is shown here). This finding supports the hypothesis of TTS, as shear is responsible for $\Psi$ splitting. ${ }^{[28]}$ This conclusion is further supported by the numerical results of Sawada et al., ${ }^{[29]}$ Bambach et al. ${ }^{[30]}$ and Mishra et al. [14]

Up to this point, the formation of residual stresses in SPIF is basically based on phenomenological considerations. Generally, residual stresses are elastic stresses which remain in the plastic deformed material after the load has been removed. As the material's strength goes up because of increased work hardening, the associated increased yield strength enables higher residual stresses as these are elastic stresses. ${ }^{[31]}$ Therefore, the influence of TTS is noticeable by enabling higher residual stresses due to an increase in the yield strength (FWHM). There is no active impact on the magnitude of the residual stresses because TTS does not influence the plane, near-surface residual stress state, discussed in this study $(\sigma 11, \sigma 22)$, as could be shown with the example of the shear specimen (see Table II). ${ }^{[22]}$ The origin and magnitude of the residual stresses presented in this study are explicitly discussed in chapter 4.2 .

The ascertained correlation between the number of step-down increments and TTS could not be observed for large cone geometries to the extent described (Figure 8(b)). Even for a higher number of forming steps $\sum \Delta z$ than the parameters used for small truncated cone geometries, only weak TTS influences on the intensity distribution in the 222 pole figure on the near-surface tool side for the large cone geometry were noted. In contrast, pronounced influences of TTS have been demonstrated for small cone geometries with an increased number of forming steps (see Figure 8(a)).

As the distance rises to the tool contact area, decreasing TTS influences on the intensity distributions in the 222 pole figures were observed. The 220 pole figures on the tool-averted side utilized for these samples illuminate another effect, which is bending. Therefore, the forming process is affected by varying forming mechanisms over the sheet thickness, discussed in more detail in chapter 4.2 for the tool-averted side.

\section{B. Bending and Stretching}

The analysis carried out points up a dependence of the occurring bending and stretching deformation on the magnitude of the vertical step-down increments $\Delta z$ and specimen geometry used. In that respect, the influence of the bending mechanism during the SPIF process was observed to be more pronounced at the near-surface tool-averted side of incrementally formed sheets (Figs. 9 and 10). The greater distance to the tool contact area means that the influence of bending on the spatial orientation distribution of the crystallites gets stronger, which results in pronounced bending-induced plasticization on the tool-averted side. The influence of bending on the spatial orientation distribution of the crystallites can be seen in the new formation of intensity maxima on the equatorial plane in the 220 pole figures of the reference bending state (Figure 5(c)). Based on this observation the changed orientations of crystallites on the tool-averted side due incremental forming using larger step-down increments $\Delta z$ can be obviously attributed to the bending mechanism (see Figure 9(a)).

This way, the impact of bending on the work-hardening state of the incrementally formed specimen is explained in the following passage. Starting with the reference bend specimen, an increased FWHM maximum of $0.72 \mathrm{deg}$ on the tool-averted side compared to $0.62 \mathrm{deg}$ on the tool side was determined (see Table II). This observation concludes that the work-hardening state on the tool-averted side of the bend specimen is more pronounced compared to the tool side. This observation is due to a larger plastic deformation on the tool-averted side of the sheet caused by bending. This hardening state corresponds to a specimen formed by SPIF with an increased step-down increment $\Delta z$ and decreasing number of forming steps, e.g., single-stage formed linear grooves $\left(\sum \Delta z=1 ; \Delta z=10 \mathrm{~mm}\right.$ ) (see Table III). In terms of the development of work hardening discussed in the literature ${ }^{[32]}$ bending is an important factor for the work-hardening state on the tool-averted side's incrementally formed sheets.

With a decreasing step-down increment $\Delta z$ size, the bending mechanism's influence on the intensity distribution in the 220 pole figure decreases significantly (see Figure 9).

Despite lower bending-induced plastic deformation, the rising work hardening with a growing number of forming steps $\sum \Delta z$ on the tool-averted side of the specimens (see Table III) is due to an increasing TTS 
influence on the forming process (see Section IV-A). This statement is supported by the 220 pole figures shown in Figs. 9 and 8. There, the initial rolling texture on the tool-averted side is progressively influenced by TTS. This is inferred from the distortion of the rolling texture, which is attributed to shear by experimental investigations of Dirras et al.. ${ }^{[33]}$ The greater impact of TTS over the entire sheet thickness is due to a reduction in sheet thickness with a growing number of forming steps $\sum \Delta z .^{[22]}$ This leads to a hardening of the sheet despite a decreasing bending influence even on the tool-averted side (see Table III). However, since the influence of TTS on the tool-averted side is lower compared to the tool side, no highly pronounced TTS-induced intensity distributions in the 222 pole figures and forming-induced work hardening on the tool-averted side were detected in specimens manufactured with a multitude of forming steps (see Section 4.1). This way, using an increased number of forming steps $\sum \Delta z$, the magnitude of work hardening on the tool-averted side is lower than on the tool side of the sheet.

This finding is pointed out by the FWHM using the example of plate geometry $\left(\sum \Delta z=20\right.$, see Table III). In agreement with the TTS detection by the texture analysis (see Figure 5), the FWHM on the tool side with $0.81 \mathrm{deg}$ is more strongly pronounced than that on the tool-averted side with $0.77 \mathrm{deg}$. Due to the direct correlation of the FWHM with the work-hardening state of formed metallic materials, a stronger hardening of the tool side can be concluded. In agreement with Mishra et al., ${ }^{[14]}$ TTS is an essential forming mechanism in SPIF.

With the help of the spatially resolved investigation presented in this study, it could be shown that bending is also a dominant forming mechanism in SPIF using increasing step-down ratios. This spatially resolved insight expands the understanding of the process where the step-down ratio $\Delta z$ is an essential factor concerning the dominant forming mechanisms in SPIF.

The presented intensity distribution for large cone geometry in the 220 pole figure elucidates a significant influence of plastic deformation by bending on the tool-averted side (see Figure 10). Although using an identical step-down increment $\Delta z$ of $3.75 \mathrm{~mm}$ for small and large truncated cone geometries, the intensity distribution affected by bending can only be observed in the 220 pole figure of the large cone geometry (see Figure 10). Thus, depending on the component height, an obvious change of the dominant forming mechanism takes place. For the large truncated cone geometry, the bending mechanism plays a decisive role in the forming process, whereas for small truncated cones, TTS is a dominant factor during forming. The increase in the described bending influence on plastic forming, particularly for large cone geometries, is due to increased bending moment with growing component size. Besides the larger cones of the increased bending moment, TTS is reduced because of less over-rolling of the near-surface material due to larger distances between the tool paths. These aspects establish the change in the dominant forming mechanism from TTS to bending deformation on the near-surface tool-averted side for large cone geometries.

The discussed relationship between the step-down ratio and the forming mechanisms, according to Sebastiani [5] and Li et al., [34] strongly depends on the component geometry used. Sebastiani ${ }^{[5]}$ predicts that the shear influence decreases with an increasing number of forming steps used. Considering the results of this work, this finding is only consistent for larger total forming heights. Li et al. ${ }^{[34]}$ conclude the opposite from their presented experiments. Therefore, Li et al. [34] ascertain a higher influence of shear on the forming process with an increasing number of step-down increments. This is in agreement with the observation for smaller truncated cone geometries shown in this study.

It was experimentally proven that an increased step-down increment $\Delta z$ also causes a more pronounced global stretching (see Figure 11). These experimental findings regarding bending and stretching verify the statements of Silva et al., ${ }^{[1]}$ Emmens and Boogaard [3] that the SPIF-process consists of a combination of bending and stretching referred to as bending under tension.

The insights obtained from the depth-resolved texture analysis of this work indicate that using smaller stepdown increments (respectively, a higher number of forming steps) strengthens TTS's influence. This experimental proof is fundamental for understanding the increased forming limits of SPIF because the TTS is considered a significant cause of the enhanced formability in SPIF, according to Eyckens et al.. ${ }^{[35]}$ Also, smaller step-down increments reduce the bending and stretching influence during forming, which, following Maqbool et al.'s ${ }^{[7]}$ numeric studies, results in improved geometric accuracy by reducing spring-back.

The results presented in section 3.5 illustrate the generation of near-surface residual stresses during the SPIF process for a set of parameters. The residual stress states of incrementally formed specimens are characterized by residual tensile stresses at the tool contact area and residual compressive stresses at the near-surface tool-averted side (see Tables III and IV). This residual stress state is assignable to the bending mechanism.

The comparable near-surface residual stress state supports this statement to the bent specimen (see Table II) and, on the other hand, by findings of the texture analysis experiments concerning bending influences on the forming process as discussed earlier.

With an increasing magnitude of incremental stepdown $\Delta z$ and a simultaneous rise of the bending influence, the residual stresses of the formed sheet approximate in both the transverse and longitudinal direction to the residual stress state of the bent specimen, since the bending mechanism acts both transversely and longitudinal to the progressive forming tool. ${ }^{[2]}$ On the tool side, the residual tensile stresses rise perpendicular to the movement direction $\left(\sigma_{22}\right)$ with increasing magnitude of incremental stepdown $\Delta z$ to the level of the bent specimen $\left(\sigma_{22}=+130 \mathrm{MPa}\right)$. However, the drop of residual stresses on the tool side in 
longitudinal direction $\left(\sigma_{11}\right)$ occurs as the magnitude of incremental stepdown $\Delta z$ increases because of compensation of the bending-induced compressive stresses through the roll-like deformation. Right before the tool passes the gauge volume, bending-induced compressive stresses were induced on the tool side. After the tool has moved on and passed the gauge volume, the near-surface elongation caused by the tool movement described in section 4.1 (see Figure 12) partially compensates the bending-induced compressive stresses. After removing the load, the attenuated bending-induced compressive stresses in the rolling direction on the tool side relax to weakly pronounced residual tensile stresses.

Hence, higher residual tensile stresses on the tool side perpendicular to the direction of tool movement were determined than in the longitudinal direction, as can be seen from the example of the single-stage formed linear groove (see Table III $\Delta z=10 \mathrm{~mm} ; \sum \Delta z=1$ ).

On the tool-averted side the tool contact described in previous sentences, according to which both residual stress components $\left(\sigma_{11}\right.$ and $\left.\sigma_{22}\right)$ show high bending induced residual compressive stresses, is missing (see Table III). Furthermore, by increasing the bending moment, higher residual compressive stresses were determined on the near-surface tool-averted side perpendicular to the direction of tool movement for large cones $\sigma_{22}=-180 \pm 15 \mathrm{MPa}$ compared to small cones $\sigma_{22}=-130 \pm 20 \mathrm{MPa}$ using a constant incremental step-down size during manufacturing. Based on the texture analyses (see Figure 10), the described increase in residual compressive stresses for larger cone geometries is related to an increasing bending influence. Generally, the level of residual stresses is caused by an increase in bending ratio, while additional TTS enables the nominal yield strength to be exceeded because of a pronounced work hardening (see Chapter 4.1). Hence, the residual stress state of formed sheets by SPIF is a combination of TTS, bending and stretching influences. ${ }^{[22]}$

\section{CONCLUSION}

In this work, the SPIF process was investigated via depth-resolved high-energy synchrotron x-ray diffraction analyzing the acting forming mechanisms during plastic forming. A conclusive discussion on roles of different deformation mechanisms during forming can be made when effects of step-down increment size and work hardening are taken into account. This requires sophisticated characterization methods. The investigations presented in this study showed that high-energy synchrotron x-ray diffraction is a suitable method for spatially resolved texture analysis of the forming mechanisms acting during the SPIF process. For the first time, the coexistence of bending, superimposed global stretching and TTS has been demonstrated using only one measuring method. Based on the detailed insights gained from the texture analysis on the main forming mechanisms acting in SPIF, it was possible to explain the origin and development of the residual stresses in SPIF for various geometries and a set of parameters.

The following conclusions can be drawn from the investigations carried out:

- The tool exerts a rolling-like loading on the near-surface tool side when the hardening state of the specimen is weak.

- With an increased number of step-down increments, TTS has been identified as the major deformation mode for linear, plate and small cone geometries.

- The occurrence of TTS is associated with a pronounced work hardening particularly in the tool contact area.

- An increased impact of the bending mechanism has been observed for high step-down increments on the near-surface tool-averted side.

- Bending causes comparatively higher work hardening on the tool-averted side of the formed parts and is favored by a high bending moment.

- One of the key origins of residual stresses during SPIF is the plastic deformation caused by bending and stretching.

- Bending has the most pronounced effect on the magnitude of residual stresses.

- The association of TTS with a pronounced work hardening enables higher residual stresses due to an increase in the yield strength.

The residual stress state is affected by the TTS, the bending mechanism and stretching. The influence of the mechanisms on the residual stress state is dependent on the process parameters, the geometry to be formed and the hardening state of the sheet metal.

The acquired knowledge gained from the depth-resolved texture analysis of incrementally formed sheets by SPIF enables an extensive understanding of the forming process with the possibility of manufacturing sheet metal products in an application-optimized way in the future. Several authors attribute the immediate effects of the acting forming mechanisms to the properties of incrementally formed sheets. Eyckens et al. ${ }^{[35]}$ and Allwood et al. ${ }^{[8]}$ present a correlation between the TTS and the increased forming limits of the SPIF process compared to conventional cold-forming methods. Maqbool et al. ${ }^{[7]}$ establish a direct relationship between the forming mechanisms and the geometric accuracy of the sheet metal products. Thus, the authors conclude with a numerical analysis in which the sheet metal products' geometrical accuracy decreases with increasing bending influence due to a stronger springback. Maaß et al. ${ }^{[36]}$ investigated the influence of residual stresses on the lifetime of components under load. The authors observed that high bending-induced tensile residual stresses accelerate the formation of cracks and their propagation under load. In summary, the depth-resolved texture analysis for numerous parameters presented in this article allows the identification of the forming mechanisms which enable the forming in the 
SPIF process. This way, the results show, among others, that using a smaller step-down increment allows the extension of formability caused by an increased TTS influence ${ }^{[35]}$ and improves the geometric accuracy by decreasing spring-back. ${ }^{[7]}$

\section{ACKNOWLEDGMENT}

The authors acknowledge Prof. Dr.-Ing. Dr.-Ing. E.h. A. Erman Tekkaya (Institute of Forming Technology and Lightweight Components, TU Dortmund University) for initiation of the project, on which this research is based. The authors would like to thank Marlon Hahn and Fabian Maaß for manufacturing the specimens and proofreading the manuscript. Furthermore, the authors acknowledge Felix Schmeiser and Erwin Krohmer for facilitating the synchrotron investigations at the Deutsches-Elektronen-Synchrotron, Hamburg, Germany.

The authors would like to thank the German Research Foundation (DFG, Deutsche Forschungsgemeinschaft) for funding the research project 372803376.

\section{DATA AVAILABILITY}

The raw/processed data required to reproduce these findings will be made available on email request.

\section{CONFLICT OF INTEREST}

The authors declare that they have no known competing financial interests or personal relationships that could have appeared to influence the work reported in this paper.

\section{FUNDING}

Open Access funding enabled and organized by Projekt DEAL.

\section{OPEN ACCESS}

This article is licensed under a Creative Commons Attribution 4.0 International License, which permits use, sharing, adaptation, distribution and reproduction in any medium or format, as long as you give appropriate credit to the original author(s) and the source, provide a link to the Creative Commons licence, and indicate if changes were made. The images or other third party material in this article are included in the article's Creative Commons licence, unless indicated otherwise in a credit line to the material. If material is not included in the article's Creative Commons licence and your intended use is not permitted by statutory regulation or exceeds the permitted use, you will need to obtain permission directly from the copyright holder. To view a copy of this licence, visit http://creat ivecommons.org/licenses/by/4.0/.

\section{REFERENCES}

1. M.B. Silva, M. Skjoedt, A.G. Atkins, N. Bay, and P.A.F. Martins: J. Strain Anal. Eng. Des., 2008, vol. 43, pp. 15-35.

2. F. Maaß, M. Hahn, A. Tekkaya, M. Dobecki, A. Poeche, K. Brömmelhoff, and W. Reimers: Prod. Eng., 2019, vol. 13, pp. $149-156$.

3. W.C. Emmens and A.H. van den Boogaard: J. Mater. Process. Technol., 2009, vol. 209, pp. 3688-95.

4. W.C. Emmens and A.H. van den Boogaard: KEM, 2007, vol. 344, pp. 519-26.

5. G. Sebastiani: Dissertation, TU Dortmund University, 2016.

6. T.J. Kim and D.Y. Yang: Int. J. Mech. Sci., 2000, vol. 42, pp. $1271-86$.

7. F. Maqbool and M. Bambach: Int. J. Mech. Sci., 2018, vol. 136, pp. 279-92.

8. J.M. Allwood, D.R. Shouler, and A.E. Tekkaya: KEM, 2007, vol. 344 , pp. $621-28$.

9. F. Maaß, M. Hahn, and A.E. Tekkaya: Metals, 2020, vol. 10, p. 656.

10. T. McAnulty, J. Jeswiet, and M. Doolan: CIRP J. Manuf. Sci. Technol., 2017, vol. 16, pp. 43-54.

11. R. Ray: Acta Metall. Mater., 1995, vol. 43, pp. 3861-72.

12. G. Wassermann and J. Grewen: Texturen Metallischer Werkstoffe. Springer, Berlin, 1962.

13. W. Williams: Trans. Met. Soc. AIME, 1962, vol. 224, pp. 129-39.

14. S. Mishra, K.U. Yazar, A. More, L. Kumar, R. Lingam, N.V. Reddy, and S. Suwas: Mater. Sci. Eng. A, 2020, vols. 1-15, art. no. 139311 .

15. H. Bunge, L. Wcislak, H. Klein, and S.J. Garbe: Adv. Eng. Mater., 2002, vols. 4-5, pp. 300-05.

16. B.B. He: Two-Dimensional X-ray Diffraction, Wiley, Hoboken, 2009.

17. N. Schell, A. King, F. Beckmann, T. Fischer, M. Müller, and A. Schreyer: $M S F, 2013$, vol. 772, pp. 57-61.

18. A. Hammersley: Computer program FIT2D. Reference Manual Version 3.1. Internal Report ESRF, 1998.

19. L. Lutterotti, R. Vasin, and H. Wenk: Powder Diffr., 2014, vol. 21, pp. 76-84.

20. M. Rietveld: J. Appl. Crystallogr., 1969, pp. 65-71.

21. J. Robinson, C. Truman, T. Pirling, and T. Panzner: Mechanical Stress Evaluation by Neutrons and Synchrotron Radiation, Grenoble, France - MECA SENS VIII, 2015.

22. M. Dobecki: Dissertation Technische Universität Berlin, 2020.

23. S. Li, I. Beyerlein, and M. Bourke: Mater. Sci. Eng., 2005, vol. 394, pp. 66-77.

24. W. Boas and E. Schmid: Zeitsch. Tech. Phys., 1931, vol. 2, pp. 71-75.

25. B. Scholtes: DGM Informationsgesellschaft Verlag, Oberursel, 1991.

26. J.E. Merwin and K.L. Johnson: Proc. Inst. Mech. Eng., 1963, vol. 177, pp. 676-90.

27. R. Malhotra, L. Xue, T. Belytschk, and J. Cao: J. Mater. Process. Technol., 2012, vol. 212, pp. 1573-90.

28. V. Hauk: Structural and Residual Stress Analysis by Nondestructive Methods, Elsevier, Amsterdam, 1997.

29. T. Sawada, G. Fukuhara, and M. Sakamoto: J. Jpn. Soc. Technol. Plast., 2001, vol. 42, pp. 1067-69.

30. M. Bambach, G. Hirt, and S. Junk: Proceedings of the 7th International Conference on Computational Plasticity, International Center for Numerical Methods in Engineering, 2003, pp. 671-74.

31. N. Svensson: J. Mech. Eng. Sci., 1966, vol. 8, pp. 162-72.

32. H. Jack, Z. Marciniak, and J. Duncan: Mechanics of Sheet Metal Forming, Elsevier, Amsterdam, 2002.

33. G. Dirras, L. Duval, and W. Swiatnicki: Mater. Sci. Eng., 1999, vol. 263, pp. 85-95.

34. Y. Li, W.J. Daniel, Z. Liu, and H. Lu: J. Mater. Process. Technol., 2015, vol. 221, pp. 100-11. 
35. P. Eyckens, A. Bael, and P. Van Houtte: Int. J. Plast., 2009, vol. 25, pp. 2249-68.

36. F. Maaß, M. Dobecki, M. Hahn, W. Reimers, and A.E. Tekkaya: Proceedings of the Contributed Papers from Materials Science and Technology, vols. 1176-1182, Portland, OR, USA, 2019.
Publisher's Note Springer Nature remains neutral with regard to jurisdictional claims in published maps and institutional affiliations. 